\title{
A NEW DYNAMIC SCHEDULING FOR BATCH PROCESSING SYSTEMS USING STOCHASTIC UTILITY EVALUATION FUNCTION
}

\author{
Hongsuk Park \\ Amarnath Banerjee \\ Texas A\&M University \\ Dept. of Industrial and Systems Engineering \\ 3131 TAMUS \\ College Station, TX 77843, U.S.A.
}

\begin{abstract}
In long production cycles, the earliness and tardiness weight (utility) of products vary depending on the time. It is necessary to reflect the weight of products for earliness and tardiness at decision epochs to decide on the optimal strategy. This research demonstrates the use of Stochastic Utility Evaluation (SUE) function approach to optimize system performance using multiple criteria. In addition, this research explores how SUE function using stochastic information can be derived and used to strategically improve existing approaches. SUE function for earliness and tardiness is used in an existing model to develop a triobjective problem. Typically, this problem is very complex to solve due to its trade-off relationship. However SUE function makes it relatively easy to solve the tri-objective problem since SUE function can be incorporated in an existing model. It is observed that SUE function can be effectively used for solving a tri-objective problem.
\end{abstract}

\section{INTRODUCTION}

The semiconductor industry has continued to be an important field of research in recent years because of the widespread use of integrated circuits (IC) in devices ranging from personal computers to high-tech electronics. Due to the growth of the semiconductor market, research in IC fabrication technology and methodology is steadily expanding. Wafer production is a very complex process with a long production cycle; and much of the complexity results from batching, time-step, and sequencing problems at several stages of the cycle. The majority of problem-solving methods attempt to minimize production attributes such as cycle time, earliness and tardiness (Mathirajan and Sivakumar 2006). Most often, the batch process itself is the bottleneck, particularly since competing factors come into play for the important decision to run a partial batch versus waiting for future arrivals to form a full batch.

On-time delivery and rapid production of wafers are the two critical factors affecting the retention of customers and influencing the performance of manufacturer. Diffusion furnaces are the most commonly used batch processors in semiconductor production. Yet while they can handle a batch of products concurrently, their processing times are too slow compared to serial processors that handle one product at a time. This research analyzes forthcoming performance-enhancing measures in the decision-making phase of batch processing that aid in controlling batch processors. At the decision epoch, most models use a static weight for product type; however, if earliness and tardiness are considered at the decision point, the dynamic weight for product type needs to be estimated according to stochastic information in the long-run control of a batch processor and multiple product types. 


\section{Park and Banerjee}

The purpose of this research is to develop a SUE function to provide the dynamic weight for product type at each decision point. The focus is on performance improvement considering multi-criteria with the use of SUE function in existing model.

This paper is organized into six sections. Section 1 introduces the research problems. In the next section, past research studies relevant to dynamic control of batch process systems are reviewed. In Section 3, the SUE function is introduced and the procedures to derive it for tardiness only, earliness only, and both earliness and tardiness are presented. Section 4 discusses how to apply the SUE function to existing approaches to solve tri-criteria (cycle time and earliness/tardiness) problem. The simulation results for benchmark strategies such as NACH-SUE, MBS-SUE, no idle and full batch are analyzed in section 5. Section 6 discusses contributions of this research and suggests topics for additional research in the future.

\section{BACKGROUND AND LITERATURE REVIEW}

There is limited amount of research studies in cited literature to use the utility value to solve strategic decision problem in dynamic control of batch processing system. In an earlier paper, Park and Banerjee (2010) explored how utility can be used to strategically control Minimum Batch Size (MBS) model for a single product. However, there is considerable work done without utility value that attempts to analyze the performance of such systems using performance metrics such as cycle time and earliness/tardiness.

Batch processing has been traditionally studied as a queuing problem. Bailey (1954) was probably the first to address a bulk service queuing system for single arrival/single server system with Poisson process and service in batches. A conventional batch processing strategy is Minimum Batch Size (MBS) which is considered as one of the general methods for processing batches. Deb (1976) showed a dynamic programming approach to obtain the optimized MBS strategy to minimize expected cost. Neuts (1967) considered optimizing a batch service queue with Poisson arrivals in case of a single lot using MBS rule. In this method, when the number of lots in the queue exceeds the predetermined MBS value, a batch is processed. Neale and Duenyas (1997) described the mixture of product type case where distributions of arrivals for each lot are different and a large number of different types can be batched together. Near-future arrival information is assumed to be available in order to make a decision, a decision epoch is chosen at points when batch processor becomes idle or an arrival occurs while the batch processor is idle. Duenyas and Neale (1997) suggested an optimal control limit method for a single batch process for two types of lots. In this paper, heuristic control policies to solve for larger number of lot types are proposed. Glassey and Weng (1991) showed the first look-ahead batch control policy, which is called as DBH using mean waiting time of lots just before a batch processor as the performance measure. Fowler, Phillips, and Hogg (1992) provided a Next Arrival Control Heuristic (NACH), which integrated a rolling horizon approach. $\mathrm{NACH}$ uses only the next arrival time, and determines whether it is more efficient to start the batch process at the next arrival time. The process of the decision making is repeated when this arrival occurs. In their later research, NACH approach was extended for multiple processors by Fowler, Hogg, and Phillips (2000).

Considering cycle time and due date criteria, Mönch et al. (2005) have provided two different decomposition approaches for the given NP-hard problem in order to minimize total weighted tardiness on parallel machines with incompatible families and jobs with unequal ready times. The first approach fixes the batches, assigns them to the machines using GA, and sequences the batches on each machine. The second approach assigns jobs to machines using a GA, figures the batches for each individual machine for the assigned jobs, and sequences these batches.

Reichelt and Mönch (2006) have focused on minimizing makespan and total weighted tardiness on multiple batch processors. Their approach follows the three-stage (batching, assignment and sequencing) algorithm developed by Mönch et al. (2005). The adaptation occurs in the batch assignment stage (GAbased method), Non-dominated Sorting Genetic Algorithm II (NSGA-II) is utilized to find the Paretooptimal solutions, and a local search method is utilized to improve the Pareto-optimal solutions. Mason (2007) has used NSGA-II to improve on time delivery, decrease cycle time variation, and decrease violation of the timer to minimize recirculation. Ganesan, Gupta, and Iyer (2004) have proposed scheduling for 


\section{Park and Banerjee}

the batch processors to minimize mean cycle time and maximum tardiness. According to this research, each decision is made within the short-term future and the outcomes with respect to the criteria are evaluated. It shows Pareto-optimal decision to be obtained and provided to the decision-maker as the Paretooptimal boundary. For the NP-hard problem of $n$ independent jobs with due dates and sequencedependent setup times, Gupta and Sivakumar (2005) have utilized a Pareto-optimal approach to minimize average cycle time, average tardiness, and to maximize machine utilization. First, they used a discrete event simulation approach and then compromise programming approach at each decision point in simulated time.

There are a few research studies using utility evaluation function in batch processing that is typically found in semiconductor manufacturing system. Clark (1990) introduced time-value function, which considers the relative utility values of the activities that they have, in scheduling dependent real-time activities.

While various research studies to solve single or bi-criteria problem have been introduced in this section, there are scant research studies which consider the weight of each product type that changes over time. As discussed in section 1 , in a long production cycle time system the utility value of lots can vary for diverse factors, such as contractual penalties, change of price for product, loss of customer goodwill, surplus of lots, etc. Among the time-dependent factors, due date and storage cost, are particularly critical, since utility value can significantly vary in response to the penalties imposed for missing the due date deadline. Obtaining the utility evaluation function which reflects the accurate value at each epoch is more desirable from a business perspective. Therefore a SUE function is developed and introduced in the next section to overcome the limitation in the existing literature.

\section{STOCHASTIC UTILITY EVALUATION FUNCTION APPROACH}

In practice, the factors due date and storage cost have considerable impact on utility value. A utility evaluation function can be approximately derived from existing business information on due date that affects tardiness and storage cost that affects earliness. Clark (1990) has identified the time-utility function by considering the very simple case of time-utility function which is due date-oriented.

Park and Banerjee (2010) have used three utility evaluation functions and applied these to existing models - MBS-U and DBH-U. For ease of explanation, they make the following assumptions for utility evaluation function:

1. Utility evaluation function is a linear time utility model.

2. The due date used in time utility function is "current time," not "expected time to finish".

In other words, use the current time to obtain utility value at the point when current time is not past the due date, but expected time to finish the job is past the due date.

Their research, however, was preliminary and limited since they assumed linear utility evaluation functions. According to assumptions made for utility function, the result can vary substantially and be trivial. They did not consider due date prior to occurrence. For example, if tardiness is not considered before due date in the bi-criteria problem (cycle time and tardiness), the algorithm focuses on reducing cycle time. However, as the time approaches due date, tardiness becomes more important; thus, to reflect its importance before the due date, the utility function must have a value before due date. Finally, although they have used a stochastic process for the interarrival time, information from the distribution, such as the likelihood estimator, was not used for decision making. A stochastic utility evaluation function has been developed in this research in order to overcome these limitations.

\subsection{Introduction of the Stochastic Utility Evaluation (SUE) Function}

Most of the literature has used stochastic process for arrivals such as exponential distribution (Cerekci and Banerjee 2010; Fowler, Phillips, and Hogg 1992; Van Der Zee, Van Harten, and Schuur 2001), and for solving the bi-criteria problem, the subjective weight for tardiness has been specified by decision maker. However, in a stochastic process, the probability function which indicates the values of the rela- 


\section{Park and Banerjee}

tionship between the two variables, a random variable and independent variable, can be derived. Also, the probability function introduces the variable indicating the values within the range of a random variable and the probability as the dependent variable. Therefore, when the weight for tardiness changing over time needs to be considered, the value from the probability function can be used as the weight for tardiness. The value from a probability function can be considered as more objective than the value obtained by a decision maker. Even though the value from a decision maker is subjective, in the existing approach for solving the bi-criteria problem the weight for tardiness is still decided by decision maker, and the weight is fixed over time in their approaches. Based on the weight from a probability function, this research introduces a Stochastic Evaluation Utility (SUE) function that captures the weight for the tardiness that changes over time.

The following notations are used here for the SUE function:

$N_{j} \quad=$ Number of orders for product $j$

$D_{j} \quad=$ Due date for product $j$

$C_{t j} \quad=$ Completed jobs for product $j$ at time $t$

$a_{j} \quad=$ Number of remaining orders for product $j$ at time $t$

$W_{j}(t)=$ Weight function for product $j$ at time $t$

$F_{j}\left(t, a_{j}\right)=$ Probability function for product $j$ at time $t$ given number of remaining orders for product $j$

The SUE function for batch process control problem can be defined as follows:

$$
U_{j}(t) \quad=W_{j}(t)=f\left(F_{j}\left(t, a_{j}\right), D_{j}, N_{j}, C_{t j}\right)
$$

The objective of SUE function is to find the optimal weight for tardiness. Parameters and variables from the problem, (e.g., the percentage jobs that can be completed by due date), are used in SUE function. The SUE function $U_{j}(t)$ combines the probability to complete jobs by due date using a stochastic approach, and the other deterministic parameters: due date, number of orders, completed jobs and remaining orders. For example, assume that arrival rate of a specific job type follows exponential distribution, due date is time $T$ and number of orders is $n$. Two cases are possible. The first case estimates the probability to finish remaining jobs by due date at a decision epoch. If the probability is low, it means that there will not be enough arrivals of lots to complete all the jobs by due date. Thus the weight for tardiness should be high to complete the jobs by due date. The second case finds the ratio of remaining orders and number of possible jobs to complete them. If the ratio is high, less number of lots than orders will arrive at the batch processor by due date. Thus the SUE function can be obtained as in the first case.

Earliest Due-date (EDD) is a common strategy to prioritize different batch arrangements. When there is an available product in queue, a batch is processed according to EDD strategy. The batch which has a minimum average due-date is chosen to be processed on the machine. However, EDD strategy has a vital problem when due-date for each product is very similar and the number of remaining order for each product has a large difference. For example, if there are two products (Product 1 and 2). Due date of product 1 is just ahead of due date of product 2 and the number of products in queue is the same. However, at decision epoch, the number of remaining orders is significantly different for each product. If EDD strategy is used to choose an alternative, the decision is that the batch of product 1 is processed. However, even though due date of product 2 is later than product 1, the batch of product 2 needs to be processed since product 1 still has a chance to be on time (no tardiness) for the remaining time until due date. On the other hand, if product 2 (which has a large number of remaining orders) is not processed at decision epoch, the possibility to be on time becomes lower and tardiness becomes larger.

From this point of view, a SUE function strategy is more useful than EDD strategy since the SUE function considers due date, number of remaining orders and remaining time until due date. 


\section{Park and Banerjee}

\subsection{SUE Function for Tardiness}

Researchers have proposed various approaches and algorithms considering cycle time, tardiness and both cycle time and tardiness for solving the batch process control problem. Among these approaches, solving the bi-criteria objective optimization problem is more difficult than the single criterion objective problem, due to the trade-off between cycle time and tardiness. In order to solve the bi-criteria problem, they have focused on ways to combine the weight for each criterion into a single criterion. However there are limitations to this approach as they do not account for changing weight for each criterion. This research modifies the existing approach using a SUE function to solve the bi-criteria problems to reflect the changing weight over time.

Genetic and Pareto-optimal approaches have been used to solve bi-criteria objective optimization problems. The latter approach combines multiple objectives into one scalar objective. Pareto optimal approaches can be categorized into weighted aggregation, minimum fractional deviation, global criterion and compromise programming. All of these use weights for all the criteria chosen by the decision-maker. The weight chosen by a decision maker can often lack objectivity. On the other hand, the use of SUE function, which is derived from stochastic information, such as expected estimation, can provide the desired objective weight.

Assume that lots arrive at a batch processor according to an exponential distribution with rate $\lambda$, number of order $N$, and due date $D$. In order to obtain the SUE function, the probability to finish jobs given remaining time by due date is considered. The probability function $F(\cdot)$ of the random variable $X$, given number of order $N$ and due date $D$ for arrival distribution at time $t$ for a single product is defined by

$$
F(t, a)=1-P\{X \leq a \mid t\}
$$

The function obtains the estimated probability that random variable $X$ takes on a value that is less than or equal to number of remaining orders by due date. If a lower probability is estimated, the probability not to complete jobs by due date is higher, which implies that tardiness increases. Therefore, this property provides weights for tardiness at time $t$ when the SUE function is plugged into the cycle timebased approach to solve bi-criteria objective problems.

If there will be enough arrivals to complete jobs by due date, the algorithm for bi-criteria problem needs to focus on minimizing cycle time since the probability of not completing jobs is very low. In this case, the weight for tardiness can be the value of 1 and the algorithm is exactly the same as cycle time based approach. However, as time approaches due date, the probability not to complete jobs increases. Therefore the weight for tardiness increases by the amount proportional to increased probability.

Now assume that lots arrive at a batch processor according to an exponential distribution with rate $\lambda$. Figure 1 shows a specific example where as time approaches due date (100 time units), the probability to complete jobs by due date decreases.

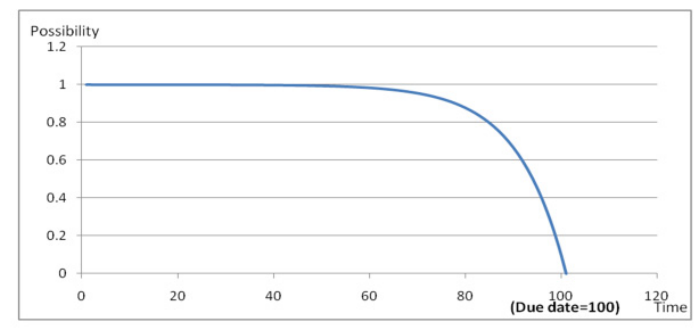

Figure 1: Time-probability function

On the other hand, as time approaches due date, the weight for tardiness increases since tardiness must be considered more than cycle time in order to complete jobs by due date. Figure 2 shows a specific instance of the weight change over time and assumes the formula 


$$
\begin{aligned}
& W(t)=1, \text { where } F(t, a)=1 \\
& W(t)=2-F(t, a), \text { where } F(t, a)<1
\end{aligned}
$$

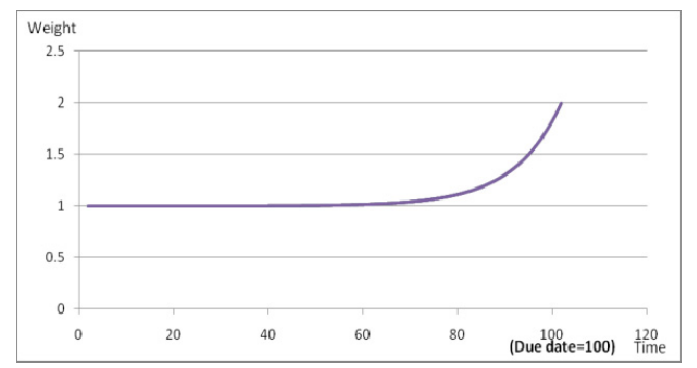

Figure 2: Time-weight function for tardiness

The applicable tardiness value can be plugged into existing approaches for minimizing cycle time in order to solve the bi-criteria objective (cycle time and tardiness) problem.

\subsection{SUE Function for Earliness and Tardiness}

The SUE function for earliness can also be derived from following the procedure for tardiness described above; however, earliness occurs far from due date. When earliness is considered in the probability function, the JIT scheduling is important. In order to acquire the JIT schedule, the minimum period when number of arrival is the same as number of orders is set up.

After due date, earliness does not need to be considered since just after completing jobs, products departs from the current system to the next. Thus, in this case, the weight for earliness is the value of 1 , which is the base value. On the other hand, the weight for the earliness is increasing as time is further from due date since time in storage is increasing.

In practice, the tardiness cost such as contractual penalty and earliness cost such as storage cost can be obtained. Therefore the ratios of the comparative weights for earliness and tardiness are used to get the probability and SUE function for the earliness and tardiness.

Now assume that lots arrive at a batch processor according to an exponential distribution with rate $\lambda$. As time approaches due date, the probability to complete jobs by due date decreases (see Figure 1). On the other hand, as time approaches due date, the weight for earliness decreases as a result of a decrease in storage cost. Figure 3 shows an example of the weight change of earliness over time and assumes the formula (the costs for tardiness include contractual penalty and loss of consumer's good will; the cost for earliness includes storage cost).

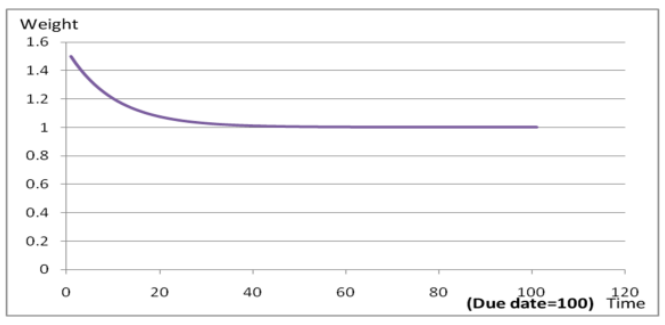

Figure 3: Time-weight function for earliness

$$
\begin{array}{lll}
r & =\text { cost for earliness } / \text { cost for tardiness } \\
W(t) & =1, & \text { where } F(t, a)=0 \\
W(t) & =1+r^{*} F(D-t, a), & \text { where } l>F(t, a)>0
\end{array}
$$


$W(t)=1+r, \quad$ where $F(t, a)=1$

Figure 4 illustrates a SUE function to solve the tri-criteria objective problem and uses the formula

$W_{E T}(t)=W_{E}(t)+W_{T}(t)-1 ; \quad$ where

$W_{E}(t)=$ Weight for earliness

$W_{T}(t)=$ Weight for tardiness

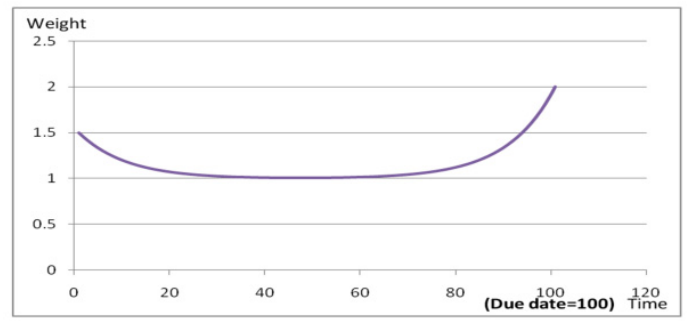

Figure 4: Time-weight function for earliness/tardiness

As mentioned earlier, there is limited research for solving the tri-criteria objective problem including cycle time, earliness and tardiness. However, the value from a SUE function (for both earliness and tardiness) can be plugged into existing approaches that minimize cycle time only.

\section{APPLICATION OF SUE FUNCTION TO THE TRI-CRITERIA BATCH PROCESS CONTROL PROBLEM}

In practical applications, a decision-maker considers multiple criteria. Due to complexity of problems and the trade-offs among criteria, few research studies have considered more than two criteria. Mathirajan and Sivakumar (2006) have reviewed and categorized the research on batch process control problems into one criterion (cycle time, tardiness, average number of jobs, etc.) and bi-criteria (cycle time and tardiness, and earliness and tardiness). There is limited research on tri-criteria including cycle time, earliness and tardiness. This section shows how to apply the SUE function to the existing models for tri-criteria objective problems.

\subsection{Modification of the Benchmark Strategy for Tri-Criteria Problem}

Using the SUE function for earliness and tardiness, a similar procedure can be applied to the triple objective approach as follows:

\subsubsection{MBS-SUE for tri-criteria problem}

Using the SUE function for earliness and tardiness, the weighted value remains constant $(=1)$ when a job is expected on time, implying that there will be no earliness and tardiness (i.e., the SUE function has the value of "1"), since the weights for commodities are not changing. In this case, MBS-SUE behaves the same as the MBS model. Once a job is expected late or early (i.e., the weight for tardiness and earliness has the values from the SUE function), the results of the MBS-SUE approach will vary with the stochastic information of arrival such as types of arrival distribution and rate, traffic density, etc. For the multiple products case, the MBS-SUE for triple criteria is similar to the MBSX approach. When more than one type of products have greater than MBS value in the queue, the one with the longest weighted waiting time using the SUE function for earliness and tardiness is selected. When there is more than one nominee, the one with the shortest weighted processing time using the SUE function for earliness and tardiness is selected. 


\section{Park and Banerjee}

\subsubsection{NACH-SUE Approach for Tri-Criteria Problem}

Using the weight from the SUE function for earliness and tardiness, the gain and loss based on weighted value in the NACH rule is obtained, and the best epoch is decided to obtain the positive gain. For the NACH-SUE for tri-criteria problem, the SUE function for earliness and tardiness is applied to the NACH approach. Therefore the additional loss for NACH-SUE caused by waiting for the future arrival at $t_{l}$ is calculated by WArea $_{1}(t)=W_{E T}(t) \times q\left(t_{1}-t_{0}\right)$.

This area, which reflects the weighting value from the SUE function for earliness and tardiness, represents the total additional loss for the $q$ lots that are in queue at $t_{0}$. The gain caused by waiting for the future arrival at $t_{1}$ is calculated by Area $_{2}(t)=W_{E T}(t) \times q\left(t_{0}+T_{j}-t_{1}\right)$. Therefore, the net gain is given by $W N e t(t)=$ WArea $_{1}(t)-$ WArea $_{2}(t)$. Similar to the procedure for a single product case, NACH-SUE for multiple product types for earliness and tardiness mimics NACH-SUE for tardiness as follows

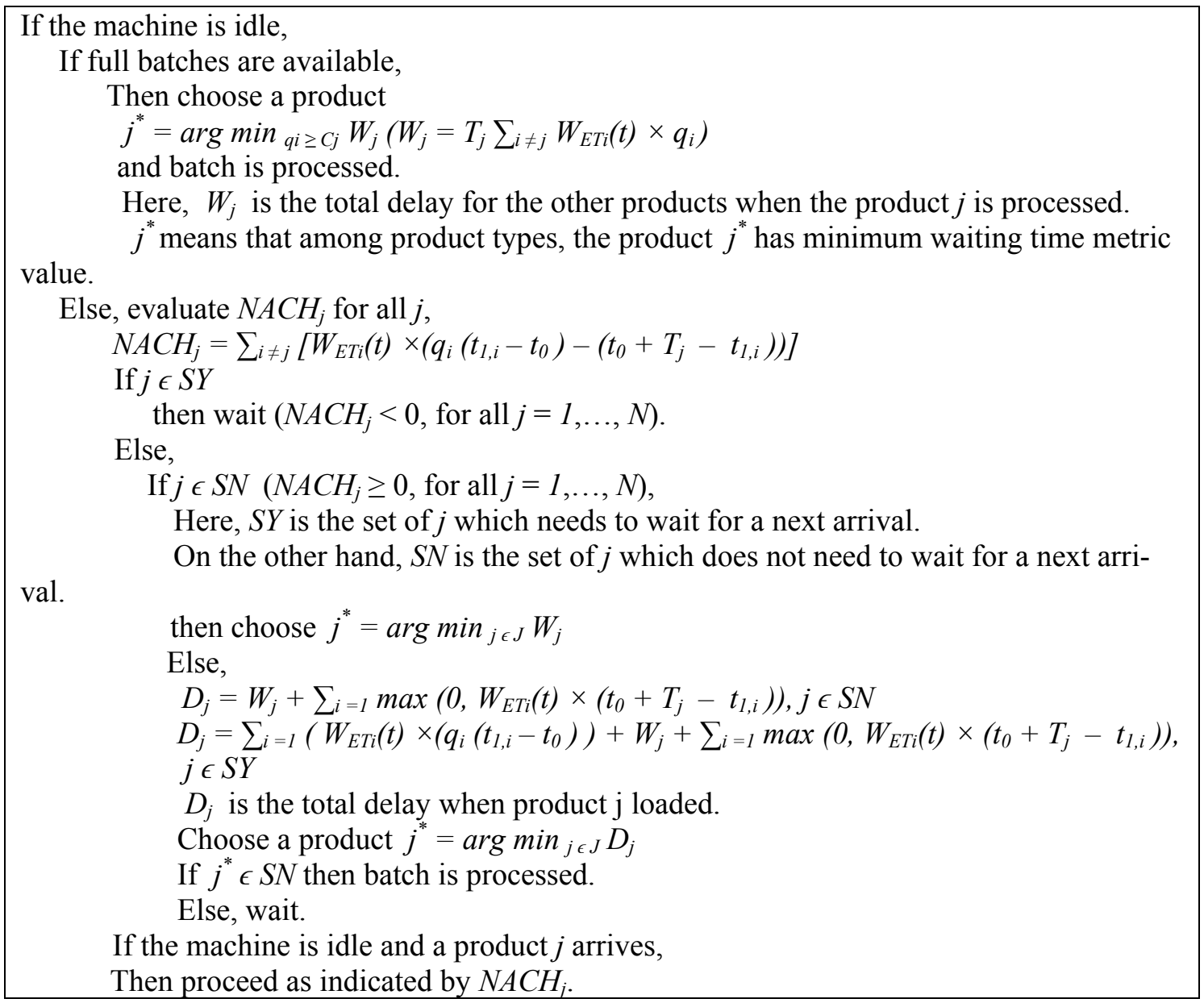

\subsubsection{Full Batch Approach for Tri-Criteria Problem}

At the decision point $t_{0}$, if only one available full batch remains in queue, the batch proceeds with the full batch. When there is more than one type of products with full batch in the queue, the type with the longest weighted waiting time using SUE function for earliness and tardiness is selected. When there is more than one nominee, the one with the shortest weighted processing time using the SUE function for earliness and tardiness is selected. 


\section{Park and Banerjee}

\subsubsection{No Idle Approach for Tri-Criteria Problem}

At the decision epoch $t_{0}$, if more than one available full batch is available, the no-idling policy is the same as the full batch policy. On the other hand, if there are only partial batches, the one with the longest weighted waiting time using the SUE function for earliness and tardiness is selected. As with the full batch policy, when there is more than one nominee, the one with the shortest weighted processing time is selected.

\section{SIMULATION RESULTS}

The performance of modification of the benchmark strategy is experimented under the same conditions for bi-criteria problem. Each of the control strategies is tested for each scenario.

A combination of the settings for simulation are:

- Control strategy: NACH-SUE, MBS-SUE, Full batch, No idle

- Simulation run length: 100,000 time units

- Number of replication: 10 times

- Warm-up period: 5,000 time units

- Number of products: 5

- Traffic intensity: $0.2,0.5,0.8$

Chaudhry and Templeton (1983) have presented the batch traffic intensity $(\rho)$ as the mean arrival rate of each products divided by the maximum batch processing rate when machine is operating at maximum capacity. The equation for traffic intensity is

$\lambda_{j}=\rho / \sum_{i=1}\left(P_{j} T_{j} / B_{j}\right)$ where

$\lambda_{j}=$ Mean arrival rate for product $j$

$\rho=$ Batch processor traffic intensity

$P_{j}=$ Product mix for product $j$

$T_{j}=$ Batch process time for product $j$

$B_{j}=$ Batch processor capacity for product $j$

The value of number of order and due date have been chosen for each scenario as

$$
N_{j}=S / \rho \times \sum_{i=1}\left(P_{j} B_{j} / T_{j}\right) \text { where } S=\text { Standard number of order, and } D_{j}=\lambda_{j} \times N_{j} \text {. }
$$

The experiment of control strategies provide 3 scenarios for the traffic intensity. Each scenario is simulated with each of the control strategies. A Pentium dual core $3.20 \mathrm{GHz}$ processor and $2 \mathrm{~GB}$ RAM is used for the scenario and Matlab 7.5 is used for the simulation code.

The performance (cycle time, earliness and tardiness) of NACH-SUE, MBS-SUE, No idle and full batch, are compared to each other. The overall performance comparison in Table 1 shows that the best performing rule is NACH-SUE. Overall performance improvements obtained by NACH-SUE are 2.48$79.25 \%$ for cycle time, $53.51-84.02 \%$ for earliness and $2.01-40.00 \%$ for tardiness, $37.96-49.14 \%$ for cycle time, $39.96-52.27 \%$ for earliness and $34.99-48.74 \%$ for tardiness, and $42.07-54.58 \%$ for cycle time, 48.50-58.25\% for earliness and 36.60-43.96\% for tardiness when compared to No idle, Full batch and MBS-SUE, respectively (see Table 1). The closest performing approach to NACH-SUE is MBS-SUE since the latter chooses the best MBS to maximize the performance. On the other hand, no idle rule and full batch rule has a lower performance since no idle rule does not allow waiting for next arrival and full batch rule is waiting for next arrival by full batch in queue. Table 1 shows the trend of performance improvements when SUE function for earliness and tardiness is used in the strategies. The overall performance improvements gained by dynamic SUE function are $20.19 \%, 12.18 \%, 12.21 \%, 16.54 \%$ for No idle, full batch, MBS-SUE, NACH-SUE respectively (see Table 2). Dynamic control strategy where utili- 


\section{Park and Banerjee}

ty value for earliness and tardiness is changing over time has significant performance improvement over static control strategy (utility value is fixed over time).

Table 1: Summary of the simulation results for tri-criteria problem: NACH-SUE is compared to the benchmark control strategies

\begin{tabular}{|c|c|c|c|c|c|c|c|}
\hline & & \multicolumn{2}{|c|}{$\mathrm{Tl}=0.2$} & \multicolumn{2}{|c|}{$\mathrm{Tl}=0.5$} & \multicolumn{2}{|c|}{$\mathrm{Tl}=0.8$} \\
\hline & & $\mathbf{P}$ & $\Delta$ & $\mathbf{P}$ & $\Delta$ & $\mathbf{P}$ & $\Delta$ \\
\hline & CT & 44.95 & 2.48 & 90.24 & 36.62 & 235.50 & 79.25 \\
\hline \multirow[t]{2}{*}{ No-idle } & E & 864.15 & 84.02 & 645.97 & 56.70 & 467.88 & 53.51 \\
\hline & $T$ & 413.32 & 2.01 & 502.94 & 15.07 & 487.48 & 40.00 \\
\hline \multirow{3}{*}{$\begin{array}{c}\text { Full } \\
\text { batch }\end{array}$} & CT & 274.24 & 47.96 & 132.09 & 49.14 & 105.09 & 37.96 \\
\hline & E & 942.13 & 52.27 & 678.93 & 51.60 & 483.47 & 39.96 \\
\hline & $T$ & 555.00 & 47.87 & 576.84 & 48.74 & 429.89 & 34.99 \\
\hline \multirow{3}{*}{$\begin{array}{l}\text { MBS- } \\
\text { SUE }\end{array}$} & CT & 44.73 & 42.07 & 67.35 & 52.12 & 81.43 & 54.58 \\
\hline & $\mathbf{E}$ & 862.53 & 56.86 & 640.96 & 58.25 & 446.51 & 48.50 \\
\hline & $\mathbf{T}$ & 408.98 & 41.45 & 429.71 & 43.96 & 349.22 & 36.60 \\
\hline \multirow{3}{*}{$\begin{array}{l}\text { NACH- } \\
\text { SUE }\end{array}$} & CT & 43.83 & - & 57.20 & - & 48.85 & - \\
\hline & E & 449.68 & - & 328.57 & - & 290.26 & - \\
\hline & $T$ & 239.44 & - & 240.82 & - & 221.41 & - \\
\hline
\end{tabular}

Table 2: Summary of the simulation results for tri-criteria problem: Approaches with the dynamic weight is compared to approaches with the static weight

\begin{tabular}{|c|c|c|c|c|c|}
\hline & & $\mathrm{TI}=0.2$ & $\mathrm{Tl}=0.5$ & $\mathrm{Tl}=0.8$ & AVG \\
\hline \multirow{3}{*}{ No-idle } & Static & 484.49 & 320.20 & 349.50 & 384.73 \\
\hline & Dynamic & 296.78 & 305.39 & 318.97 & 307.05 \\
\hline & $\Delta$ & 38.74 & 4.63 & 8.73 & 20.19 \\
\hline \multirow{3}{*}{$\begin{array}{c}\text { Full } \\
\text { batch }\end{array}$} & Static & 487.12 & 394.32 & 304.83 & 395.42 \\
\hline & Dynamic & 433.43 & 349.46 & 258.91 & 347.27 \\
\hline & $\Delta$ & 11.02 & 11.37 & 15.06 & 12.18 \\
\hline \multirow{3}{*}{$\begin{array}{l}\text { MBS- } \\
\text { SUE }\end{array}$} & Static & 346.17 & 291.37 & 257.23 & 298.26 \\
\hline & Dynamic & 294.99 & 272.51 & 217.97 & 261.82 \\
\hline & $\Delta$ & 14.78 & 6.47 & 15.26 & 12.21 \\
\hline \multirow{3}{*}{$\begin{array}{l}\text { NACH- } \\
\text { SUE }\end{array}$} & Static & 224.67 & 175.07 & 153.74 & 184.49 \\
\hline & Dynamic & 169.37 & 154.10 & 138.46 & 153.98 \\
\hline & $\Delta$ & 24.61 & 11.98 & 9.93 & 16.54 \\
\hline
\end{tabular}

Figure 5 shows that there is a steady performance improvement with increasing traffic intensity. This is due to the fact that with higher traffic intensities, the number of products in queue becomes larger and full batch is observed often. This leads to just starting the process without idling and running with full machine capacity. At low traffic intensity, performance improvement over no idle and MBS-SUE is similar to each other since the best MBS at low traffic intensity is mostly low value of MBS which is similar to no idle strategy. On the other hand, at high traffic intensity, performance improvement over full batch 


\section{Park and Banerjee}

is comparable to MBS-SUE since the best MBS at high traffic intensity is mostly high value of MBS which is similar to full batch strategy.

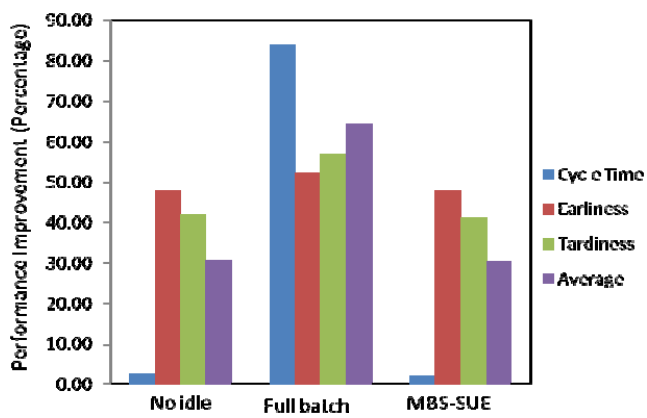

Traffic intensity $=0.2$
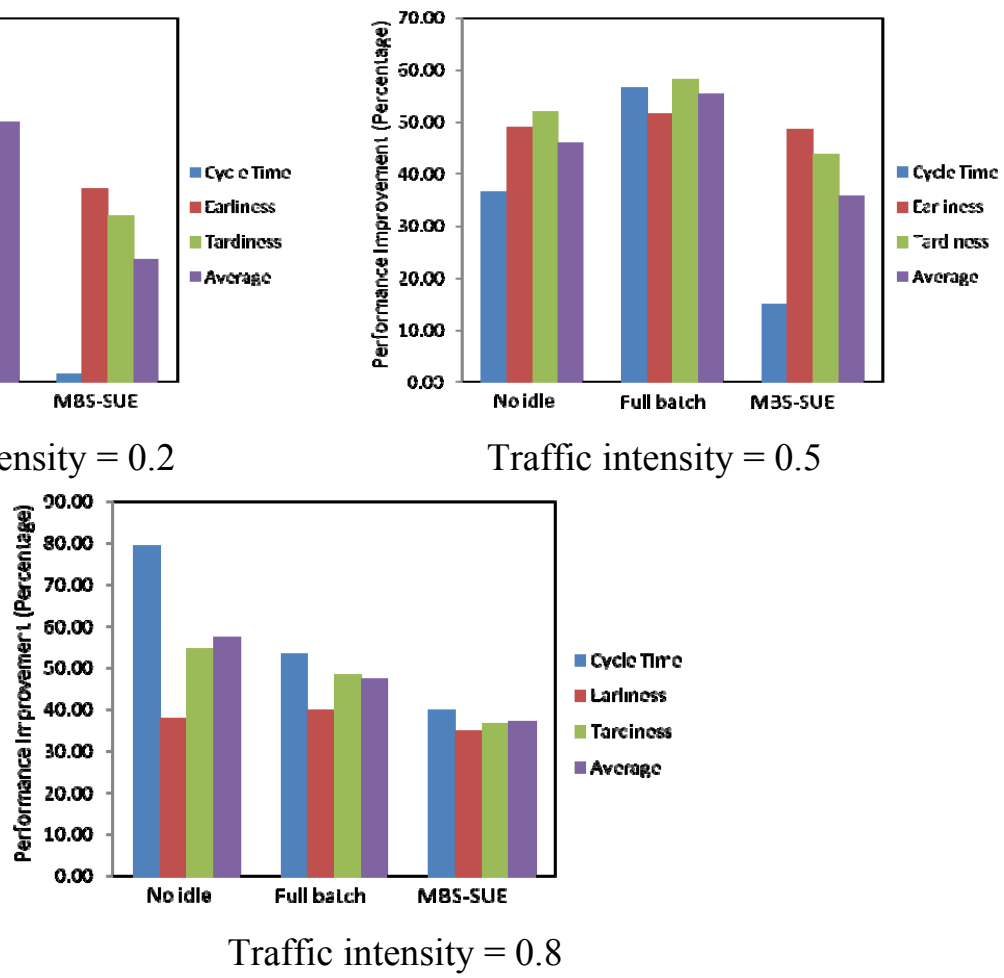

Figure 5: Performance improvements observed for NACH-SUE for tri-criteria problem with traffic intensity

\section{CONTRIBUTIONS AND FUTURE RESEARCH}

The contributions of this research are the following.

First, the main contribution of this research is to solve tri-criteria problem which has not been studied at length. This research develops a SUE function for earliness and tardiness, and then this function is applied to the benchmark strategy such as NACH-SUE and MBS-SUE in order to minimize tri-criteria (cycle time and earliness/tardiness). This shows that the SUE function considers more than two criteria simultaneously and with the use of this function, multi-objective problem can be solved with the combined strategy (SUE function and benchmark strategy).

Second, the performance of strategies for cycle time, earliness and tardiness using SUE function is improved when compared to existing model using static utility value. More importantly, it is found that the NACH-SUE strategy has the most performance improvement, and performance improvement of NACH-SUE with respect to MBS-SUE, No idle and full batch strategy is observed in the simulation scenarios.

Finally, performance improvement of NACH-SUE over the other benchmark strategies does not have significant changes with respect to number of products, machine capacity and processing time except traffic intensities among all scenarios. Performance improvements observed for NACH-SUE are in the range of $2.01-84.02 \%$, and performance differences between dynamic and static strategies are in the range of $12.18-20.19 \%$. However, among all criteria, the cycle time has increased in dynamic strategy. It shows that even though the performance for cycle time is reduced in dynamic strategy (It is to be noted that the objective of static strategy is to minimize cycle time only; however, the dynamic strategy considers tri- 


\section{Park and Banerjee}

criteria such as cycle time, earliness and tardiness), an overall performance for tri-criteria is significantly improved using the dynamic strategy.

Future research is planned along several lines. First, this approach using SUE function needs to be simulated in various settings for product diversity, product mix, machine capacity and due date. Second, the SUE function needs to be applied to various dynamic control of serial/batching processing systems. Third, there needs to be further research on estimation of the SUE function.

\section{REFERENCES}

Bailey, N. T. J. 1954. "On Queueing Processes with Bulk Service.” Journal of the Royal Statistical Society Series B-Statistical Methodology 16(1): 80-87.

Cerekci, A., and A. Banerjee. 2010. "Dynamic Control of the Batch Processor in a Serial-Batch Processor System with Mean Tardiness Performance." International Journal of Production Research 48(5):1339-1359.

Chaudhry, M. L., and J. G. C. Templeton. 1983. A First Course in Bulk Queues. New York: Wiley.

Clark, R. K. 1990. "Scheduling Dependent Real-Time Activities.” Ph.D. Dissertation, School of Computer Science, Carnegie Mellon University.

Deb, R. K. 1976. "Optimal-Control of Batch Service Queues with Switching Costs." Advances in Applied Probability 8(1): 177-194.

Duenyas, I., and J. J. Neale. 1997. "Stochastic Scheduling of a Batch Processing Machine with Incompatible Job Families." Annals of Operations Research 70:191-220.

Fowler, J. W., D. T. Phillips, and G. L. Hogg. 1992. "Real-Time Control of Multiproduct Bulk-Service Semiconductor Manufacturing Processes." IEEE Transactions on Semiconductor Manufacturing 5(2): 158-163.

Fowler, J. W., G. L. Hogg, and D. T. Phillips. 2000. "Control of multiproduct bulk server diffusion/oxidation processes. Part 2: multiple servers." IIE Transactions 32(2):167-176.

Ganesan, V.K., A. K. Gupta, and S.A. Iyer. 2004. "Bi-objective Schedule Control of Batch Processes in Semiconductor Manufacturing." In Proceedings of IEEE Conference on Robotics, Automation and Mechatronics 2:1077-1082.

Glassey, C. R., and W.W. Weng. 1991. "Dynamic Batching Heuristic for Simultaneous Processing." IEEE Transactions on Semiconductor Manufacturing 4(2): 77-82.

Gupta, A. K., and A. I. Sivakumar. 2005. "Single Machine Scheduling with Multiple Objectives in Semiconductor Manufacturing." International Journal of Advanced Manufacturing Technology 26(9-10): 950-958.

Mason, S. J., M. E. Kurz, L., M. E. Pfund, J. W. Fowler, and M. Pohl. 2007. "Multi-objective semiconductor manufacturing scheduling: A random keys implementation of NSGA-II" In Proceedings of IEEE Symposium on Computational Intelligence in Scheduling, 159-164.

Mathirajan, M., and A. I. Sivakumar. 2006. "A Literature Review, Classification and Simple MetaAnalysis on Scheduling of Batch Processors in Semiconductor." International Journal of Advanced Manufacturing Technology 29(9-10): 990-1001.

Mönch, L., H. Balasubramanian, J. Fowler, and M. Pfund. 2005. "Heuristic Scheduling of Jobs on Parallel Batch Machines with Incompatible Job Families and Unequal Ready Times." Computers \& Operations Research 32(11):2731-2750.

Neale, J. J., and I. Duenyas. 2003. "Control of a Batch Processing Machine Serving Compatible Job Families." IIE Transactions 35(8): 699-710.

Neuts, M. F. 1967. "A General Class of Bulk Queues with Poisson Input.” Annals of Mathematical Statistics 38(3):759-770.

Park, H., and A. Banerjee. 2010. "Utility-Based Dynamic Control of Batch Processing Systems." In Proceedings of the 2010 IEEE Conference Automation Science and Engineering (CASE), 439-444. Piscataway, New Jersey: Institute of Electrical and Electronics Engineers, Inc.. 


\section{Park and Banerjee}

Reichelt, D., and L. Mönch. 2006. "Multiobjective Scheduling of Jobs with Incompatible Families on Parallel Batch Machines." In Proceedings of Evolutionary Computation in Combinatorial Optimization Conference, Lecture Notes in Computer Science, edited by Jens Gottlieb and Günther R. Raidl, 3906: 209-221.

Van Der Zee, D. J., A. Van Harten, and P. Schuur. 2001. "On-Line Scheduling of Multi-Server Batch Operations." IIE Transactions 33(7):569-586.

\section{AUTHOR BIOGRAPHIES}

HONGSUK PARK completed his Ph.D. in Industrial Engineering from Texas A\&M University in 2011, Master's degree from TEPP (Techno-Economics Policy Program) in Seoul National University in 2005 and his B.S. degree in Weapons Engineering at Korea Military Academy in 1996. He is an active military officer and has been a helicopter pilot for 15 years. Since 2005, he worked as a Defense Technology Management Officer at Project Management Groups, ROKA Headquarters. His research interest lies in the Management of Defense Technology including Modeling and Simulation, heuristic optimization and strategic control for batch process systems. His email address is avipak03@tamu.edu.

AMARNATH BANERJEE is an Associate Professor in the Department of Industrial and Systems Engineering at Texas A\&M University. He received his Ph.D. in Industrial Engineering and Operations Research from the University of Illinois at Chicago, and a BS in Computer Science from Birla Institute of Technology and Science in India. His research interests are in modeling, simulation and visualization with applications in manufacturing and health care delivery systems. He teaches courses in manufacturing and production systems design and control, facilities planning, intelligent systems, virtual manufacturing and simulation. He is an Associate Editor of Journal of Manufacturing Systems, IIE Transactions on Healthcare Systems Engineering, and International Journal of Services, Operations and Informatics. He serves on the Advisory Council of the Rural and Community Health Institute, a part of Texas A\&M Health Science Center. He is a member of IEEE and a senior member of the Institute of Industrial Engineers (IIE). His email address is banerjee@tamu.edu. 